\title{
A CMB LENSING MASS MAP AND ITS CORRELATION WITH THE COSMIC INFRARED BACKGROUND
}

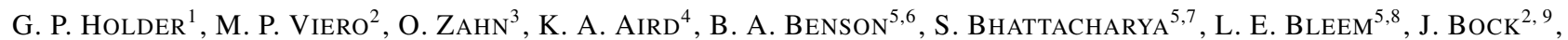
M. Brodwin ${ }^{10}$, J. E. CARlstrom ${ }^{5,6,8,11,12}$, C. L. Chang ${ }^{5,6,12}$, H-M. CHO ${ }^{13}$, A. Conley ${ }^{14}$, T. M. CRAWFORD ${ }^{5,11}$, A. T. CRITES ${ }^{5,11}$ T. DE HAAN ${ }^{1}$, M. A. DobBS ${ }^{1}$, J. Dudley ${ }^{1}$, E. M. GeORGE ${ }^{15}$, N. W. HAlverson ${ }^{16}$, W. L. Holzapfel ${ }^{15}$, S. Hoover $^{5,8}$, Z. Hou $^{17}$, J. D. Hrubes ${ }^{4}$, R. Keisler ${ }^{5,8}$, L. KNOX ${ }^{17}$, A. T. LeE ${ }^{15,18}$, E. M. LeITCH ${ }^{5,11}$, M. LUEKER ${ }^{2}$, D. LUONG-VAN ${ }^{4}$, G. MARSDEN ${ }^{19}$, D.P. MARRone ${ }^{20}$, J. J. MCMAHON ${ }^{21}$, J. MehL ${ }^{5,12}$, S. S. MEYer ${ }^{5,6,8,11}$, M. MilleA ${ }^{17}$, J. J. Mohr ${ }^{22,23,24}$, T. E. MonTROY ${ }^{25}$,

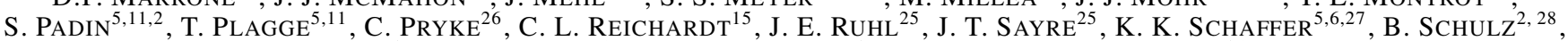

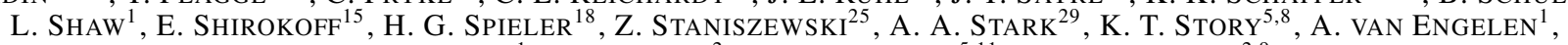

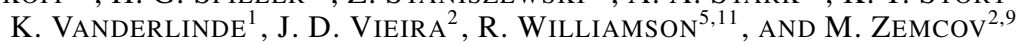

\begin{abstract}
We use a temperature map of the cosmic microwave background (CMB) obtained using the South Pole Telescope at $150 \mathrm{GHz}$ to construct a map of the gravitational convergence to $z \sim 1100$, revealing the fluctuations in the projected mass density. This map shows individual features that are significant at the $\sim 4 \sigma$ level, providing the first image of CMB lensing convergence. We cross-correlate this map with Herschel/SPIRE maps covering 90 square degrees at wavelengths of 500, 350, and $250 \mu \mathrm{m}$. We show that these submillimeter-wavelength (submm) maps are strongly correlated with the lensing convergence map, with detection significances in each of the three submm bands ranging from 6.7 to $8.8 \sigma$. We fit the measurement of the cross power spectrum assuming a simple constant bias model and infer bias factors of $b=1.3-1.8$, with a statistical uncertainty of $15 \%$, depending on the assumed model for the redshift distribution of the dusty galaxies that are contributing to the Herschel/SPIRE maps.
\end{abstract}

Subject headings: galaxies: structure—cosmic background radiation

holder@physics.mcgill.ca

${ }^{1}$ Department of Physics, McGill University, Montreal, Quebec H3A

2T8, Canada

${ }^{2}$ California Institute of Technology, Pasadena, CA, USA 91125

${ }^{3}$ Berkeley Center for Cosmological Physics, Department of Physics, University of California, and Lawrence Berkeley National Laboratory, Berkeley, CA, USA 94720

${ }^{4}$ University of Chicago, Chicago, IL, USA 60637

${ }^{5}$ Kavli Institute for Cosmological Physics, University of Chicago, Chicago, IL, USA 60637

${ }^{6}$ Enrico Fermi Institute, University of Chicago, Chicago, IL, USA 60637

${ }^{7}$ High Energy Physics Division, Argonne National Laboratory, Argonne, IL, USA 60439

${ }^{8}$ Department of Physics, University of Chicago, Chicago, IL, USA 60637

${ }^{9}$ Jet Propulsion Laboratory, Pasadena, CA, USA 91109

${ }^{10}$ Department of Physics and Astronomy, University of Missouri, Kansas City, MO 64110, USA

${ }^{11}$ Department of Astronomy and Astrophysics, University of Chicago, Chicago, IL, USA 60637

${ }^{12}$ Argonne National Laboratory, Argonne, IL, USA 60439

${ }^{13}$ NIST Quantum Devices Group, Boulder, CO, USA 80305

${ }^{14}$ Center for Astrophysics and Space Astronomy, University of Colorado, Boulder, CO, USA 80309

${ }_{15}$ Department of Physics, University of California, Berkeley, CA, USA 94720

${ }^{16}$ Department of Astrophysical and Planetary Sciences and Department of Physics, University of Colorado, Boulder, CO, USA 80309

${ }^{17}$ Department of Physics, University of California, Davis, CA, USA 95616

${ }^{18}$ Physics Division, Lawrence Berkeley National Laboratory, Berkeley, CA, USA 94720

${ }^{19}$ Department of Physics and Astronomy, University of British Columbia, Vancouver, BC, Canada V6T 1Z1

${ }^{20}$ Steward Observatory, University of Arizona, Tucson, AZ, USA 85721

${ }^{21}$ Department of Physics, University of Michigan, Ann Arbor, MI, USA 48109

${ }^{22}$ Department of Physics, Ludwig-Maximilians-Universität, 81679 München, Germany

${ }^{23}$ Excellence Cluster Universe, 85748 Garching, Germany

\footnotetext{
${ }^{24}$ Max-Planck-Institut für extraterrestrische Physik, 85748 Garching, Germany

${ }^{25}$ Physics Department, Center for Education and Research in Cosmology and Astrophysics, Case Western Reserve U niversity,Cleveland, OH, USA 44106

${ }^{26}$ Department of Physics, University of Minnesota, Minneapolis, MN, USA 55455

${ }^{27}$ Liberal Arts Department, School of the Art Institute of Chicago, Chicago, IL, USA 60603

${ }^{28}$ Infrared Processing and Analysis Center, California Institute of Technology, JPL, Pasadena, CA USA 91125

${ }^{29}$ Harvard-Smithsonian Center for Astrophysics, Cambridge, MA, USA 02138
} 


\section{INTRODUCTION}

Gravitational lensing of the cosmic microwave background (CMB) is emerging as a powerful cosmological tool. The spatial variation of the statistical properties of the CMB that is induced by gravitational lensing was first detected in crosscorrelation with radio-selected galaxy catalogs (Smith et al. 2007, Hirata et al. 2008), and subsequently detected internally in CMB maps by the Atacama Cosmology Telescope collaboration (Das et al. 2011) and the South Pole Telescope (SPT) collaboration (van Engelen et al. 2012). In Bleem et al. (2012), reconstructions of the mass distribution were found to correlate strongly with galaxy catalogs selected in both the optical and infrared bands, while Sherwin et al. (2012) showed that CMB lensing was well-correlated with quasars.

Using the CMB as the background source to study gravitational lensing by intervening structure offers several advantages over using distant galaxies: the source redshift is the same for all lines of sight, is extremely well-known, and has the highest redshift observable with electromagnetic radiation. The statistical properties of the source are wellcharacterized, and CMB maps cover areas ranging from a few hundred square degrees to the full sky. However, the single redshift for the CMB does not allow any information about the redshift distribution of the mass along the line of sight, and noise levels in CMB lensing convergence maps at current sensitivities are substantially higher than noise levels in cosmic shear measurements.

As CMB lensing is an integral along the entire line of sight, the strongest cross-correlations will be with sources that have a similarly broad extent in redshift space. As demonstrated below, and as theoretically predicted (Song et al. 2003), the cosmic infrared background (CIB) fluctuations provide an excellent match. The CIB at submm wavelengths is believed to have a substantial contribution from sources from redshifts $z \sim 0.5-3$ (Lagache et al. 2004; Amblard et al. 2011; Béthermin et al. 2011; Viero et al. 2012).

In this paper, we cross-correlate a map of the gravitational lensing convergence (proportional to the surface density) derived from SPT temperature data at $150 \mathrm{GHz}$ with maps of the submillimeter-wavelength (submm) sky at 500, 350, and 250 $\mu \mathrm{m}$ obtained with Herschel/SPIRE. By using maps rather than catalogs, as was done in previous CMB lensing crosscorrelations, we are studying emission from sources that are individually unresolved. The SPT and Herschel datasets are described in sections 2 and 3, and the results of the crosscorrelation are presented in section 4. A comparison with a simple theoretical model is presented in section 5, and we conclude with a discussion of the results.

\section{CMB MAP AND CORRESPONDING MASS MAP}

The SPT has been used to image 2500 square degrees to a depth of $\lesssim 18 \mu \mathrm{K}$-arcmin at $150 \mathrm{GHz}$, and two $\sim 100$-squaredegree fields (each subtending $1 \mathrm{~h}$ in Right Ascension and 10 degrees in Declination) within this area to a depth of $\sim 13$ $\mu \mathrm{K}$-arcmin. For this work, we use observations centered on one of those deeper fields, centered at $($ RA,DEC $)=(23 \mathrm{~h} 30 \mathrm{~m}$, $-55 \mathrm{~d} 00 \mathrm{~m}$ ), using data from both the 2008 and 2010 observing seasons; the recent CMB power spectrum measurements of Story et al. (2012) used only the data from 2008 for this field.

A CMB map is generated as outlined in Story et al. (2012). In addition, to avoid apodization effects at the edges of the field when constructing the lensing map, data from surrounding fields are used to make a single larger CMB map 17.1 degrees on a side. This map extends well beyond the region covered by Herschel data. The input CMB map is shown in the left panel of Figure 11 Adjacent fields are combined using inverse-variance weights in overlapping regions, and there is no evidence for any discontinuities at the boundaries. Point sources and massive galaxy clusters are removed using a Wiener-interpolation algorithm (van Engelen et al.|2012).

Simulated CMB maps are obtained by coadding simulated signal and noise realizations for each individual SPT field. The simulated maps are made with known input gravitational potentials, and simulated signal maps are generated using timestream-based simulations, as in Story et al. (2012). Noise realizations are obtained directly from the observations, by taking randomized combinations of the data which remove all sky signal, as detailed in van Engelen et al. (2012).

The analysis procedure is applied to both the real and simulated SPT maps. Gravitational convergence maps are generated as outlined in van Engelen et al. (2012), using the quadratic estimator method (Hu 2001; $\mathrm{Hu}$ \& Okamoto 2002). This method entails constructing a gradient-filtered map and an inverse-variance weighted map (i.e., two different filterings of the same CMB field), multiplying them together and taking a divergence. The resulting product can be shown to be an estimator for the map of the gravitational potential. The effective transfer function due to the SPT filtering was constructed by cross-correlating the derived lensing potential of the simulated maps with the lensing potential maps used to generate those simulations.

Foreground contamination of the lensing convergence maps is expected to be small: van Engelen et al. (2012) found that residual contamination of the lensing convergence map from point sources and galaxy clusters is expected to be at the level of a few $\%$. The sign of this effect is expected to be negative, such that foreground contamination acts to reduce the observed cross-correlation.

The resulting lensing convergence map is shown as contours in Figure 2. Features can be seen with significances exceeding $4 \sigma$.

\section{Herschel/SPIRE MAPS}

Submillimeter maps at 500, 350, and $250 \mu \mathrm{m}$ are created using observations with the SPIRE instrument (Griffin et al. 2003 ) aboard the Herschel Space Observatory (Pilbratt et al. 2010) obtained under an OT1 program (PI:Carlstrom). Observations were made in SPIRE fast-scan mode $\left(60 \operatorname{arcsec~s}^{-1}\right)$ and consisted of two sets of orthogonal scans covering $\sim 90 \mathrm{deg}^{2}$. The observing strategy was chosen to optimize sensitivity to large-scale signal and provide redundancy for measuring the auto-frequency power spectrum of background fluctuations.

Maps are made with SMAP, an iterative mapmaker designed to optimally separate large-scale noise from signal; the mapmaking algorithm is described in detail in Levenson et al. (2010) and updated in Viero et al. (2012). To estimate the transfer function we use the same map-making process on mock SPIRE data. For both real and mock data we make maps with 10 iterations; we have checked that the maps are adequately converged at this point. Additionally, time-ordered data (TODs) are divided into two halves and unique "jackknife" map-pairs are made. To avoid having to reproject or regrid the Herschel/SPIRE maps, we make them using the Lambert azimuthal equal-area projection (also known as zenithal equal area, ZEA), with astrometry identical to that of the SPT map, and with $30^{\prime \prime}$ pixels. 

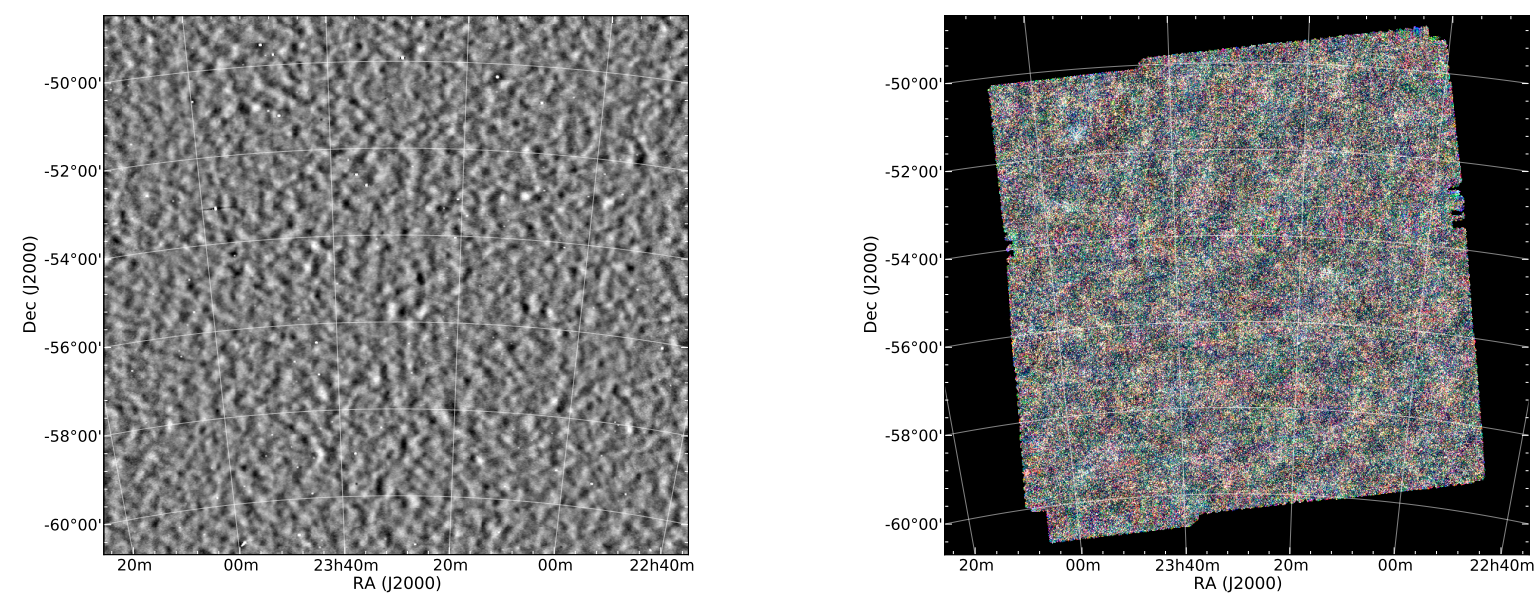

FIG. 1.- SPT $150 \mathrm{GHz}(2 \mathrm{~mm})$ temperature map (left) and Herschel /SPIRE maps (right) used for this analysis. For display purposes only the inner $\sim 60 \%$ of the SPT temperature map that was used to construct the lensing map is shown. In the right panel, (red, green, blue) correspond to $(500,350,250) \mu \mathrm{m}$.

The maps have rms instrument noise levels (per 30" pixel) of 14,10 , and $7 \mathrm{mJy}$, while the instrument effective pointspread functions are 36.6, 25.2, and $18.1^{\prime \prime}$ full widths at half maxima (FWHM) at 500, 350, and $250 \mu \mathrm{m}$, respectively, The $30^{\prime \prime}$ pixelization of the maps reduce the resolution substantially on small scales, but pixelization and instrument noise effects are not important on the scales of interest for this study. The last step, following Viero et al. (2012) is to convert the maps from native units of Jy beam ${ }^{-1}$ to $\mathrm{Jy} \mathrm{sr}^{-1}$, which is done by dividing them by the effective beam areas, 3.688, 1.730 , and $1.053 \times 10^{-8}$ steradians. Color corrections from a flat-spectrum point-source calibration have a negligible effect. The absolute calibration is accurate to $7 \%$, an uncertainty that is small compared to our statistical precision.

\section{RESULTS AND ANALYSIS}

In Figure 2, we present convergence and submillimeterwavelengths maps filtered to emphasize modes in the lensing map that have significant $(>0.5)$ signal-to-noise, allowing a by-eye comparison of the structure. Modes with $L<100$ (scales larger than $2^{\circ}$ ) have been filtered to remove scales where the timestream filtering of the submm-wave maps becomes substantial. The SPT temperature map has spatially anisotropic noise (Schaffer et al. 2011), which ultimately leads to anisotropic noise in the lensing map (van Engelen et al. 2012). This leads to a tendency for modes to be better measured when they have more horizontal structure than vertical structure.

Due to the imperfect redshift overlap, the lensing map can have features that are not in the submm maps; in particular, high-redshift structure $(z \gtrsim 3)$ will appear relatively stronger in the lensing map, while structure below $z \sim 0.5$ will be strongly suppressed in the lensing map as compared to the submm map. The submm maps are extremely wellcorrelated with each other, while the lensing map has several features that are not well-matched in any of the submm maps. Nonetheless, there are many features in common between the maps.

To compare these maps quantitatively, we use cross-power spectra, as in Bleem et al. (2012). Uncertainties are obtained by cross-correlating each submm map with lensing mass maps obtained from simulated SPT maps. We use the rms in crosspower simulated amplitudes as the rms uncertainty and as- sume a Gaussian error distribution. Cross powers are reported in Table 11, and are shown in Figure 3 .

The signal-to-noise ratio in the cross correlation is substantial: at 500, 350, and $250 \mu \mathrm{m}$ the model with no crosscorrelation is strongly disfavored relative to the best-fit lensing amplitude, with $\chi^{2}$ differences of 79,69 , and 45 , respectively. Lensing is detected in every power spectrum bin.

\section{THEORETICAL MODEL}

As a cross-check on the shape and amplitude of these spectra, we adopt the simple constant bias model used in Bleem et al. (2012), using the non-linear power spectrum at each redshift:

$$
C_{L}^{\kappa \mathrm{I}}=b \int d z \frac{d \chi}{d z} \frac{1}{\chi^{2}} W^{\kappa}(\chi) W^{\mathrm{I}}(\chi) P_{\mathrm{DM}}\left(k=\frac{L}{\chi}, z\right),
$$

where $W^{\kappa}(\chi)$ gives the redshift weighting of the mass map and $W^{\mathrm{I}}(\chi)$ is proportional to the line of sight distribution of the intensity $d I / d \chi$ (Bleem et al. 2012; Song et al. 2003). The non-linear power spectrum of the dark matter, $P_{\mathrm{DM}}$, is calculated using CAMB and Halofit, assuming the best-fit WMAP9+SPT cosmological parameters for a flat $\Lambda \mathrm{CDM}$ cos-

TABLE 1

CMB CONVERGENCE-SPIRE CROSS POWER SPECTRUM

\begin{tabular}{rrrr}
$L$ & $\begin{array}{r}C_{L}^{500} \\
(\mathrm{mJy} / \mathrm{sr})\end{array}$ & $\begin{array}{r}C_{L}^{350} \\
(\mathrm{mJy} / \mathrm{sr})\end{array}$ & $\begin{array}{r}C_{L}^{250} \\
(\mathrm{mJy} / \mathrm{sr})\end{array}$ \\
\hline 150 & $38 \pm 18$ & $101 \pm 29$ & $134 \pm 58$ \\
250 & $49 \pm 21$ & $65 \pm 30$ & $74 \pm 45$ \\
350 & $10 \pm 11$ & $18 \pm 15$ & $26 \pm 21$ \\
450 & $19.0 \pm 6.2$ & $38 \pm 10$ & $52 \pm 13$ \\
550 & $8.5 \pm 6.4$ & $15 \pm 10$ & $8 \pm 12$ \\
650 & $13.7 \pm 4.6$ & $16.0 \pm 7.8$ & $16.9 \pm 8.8$ \\
750 & $13.3 \pm 4.2$ & $17.5 \pm 5.6$ & $14.3 \pm 8.5$ \\
850 & $4.9 \pm 3.2$ & $3.5 \pm 5.4$ & $13.5 \pm 7.0$ \\
950 & $6.1 \pm 1.9$ & $9.5 \pm 3.5$ & $7.7 \pm 5.0$ \\
1050 & $6.9 \pm 1.9$ & $8.1 \pm 3.2$ & $2.1 \pm 4.6$ \\
1150 & $1.2 \pm 1.5$ & $2.9 \pm 2.4$ & $0.7 \pm 3.6$ \\
1250 & $5.7 \pm 2.2$ & $9.2 \pm 3.1$ & $11.6 \pm 4.2$ \\
1350 & $2.3 \pm 1.5$ & $5.5 \pm 2.6$ & $6.1 \pm 3.5$ \\
1450 & $4.2 \pm 1.8$ & $5.1 \pm 3.1$ & $3.8 \pm 4.1$ \\
1550 & $2.2 \pm 1.6$ & $2.4 \pm 2.9$ & $3.1 \pm 3.3$ \\
\hline \hline
\end{tabular}



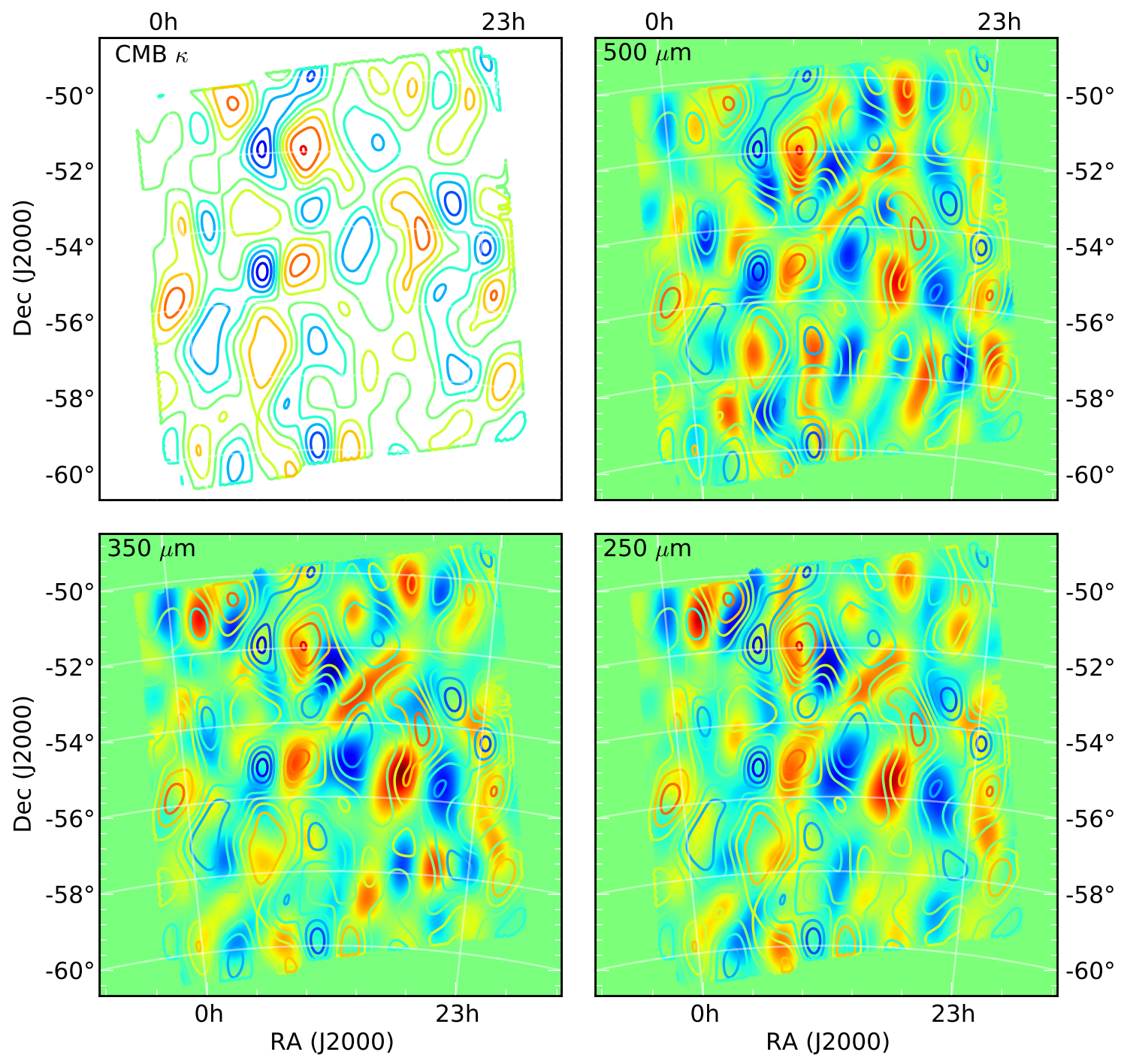

FIG. 2.- Map of the CMB lensing convergence measured with SPT data (contours in all panels) and overlaid on maps of the 500, 350, $250 \mu \mathrm{m}$ Herschel /SPIRE data (top right, bottom left, bottom right, respectively). All maps have been filtered to only show scales in the lensing map that are expected to have typical signal to noise of at least 0.5 , which suppresses all features on scales smaller than $\sim 0.5^{\circ}$. All maps have been masked by the SPIRE coverage. Lensing contours are spaced by $1 \sigma$ of noise. Red (blue) indicates regions of increased (decreased) mass or flux.

mology (Story et al.2012).

The redshift distribution of contributions to the submm background has been extensively studied in recent years, and there exist substantial disagreements between authors. We adopt two recent determinations, presented in Béthermin et al. (2011) and Viero et al. (2012) that roughly bracket expectations, to predict the cross-correlation signal. To derive this signal, we assume that the submm light traces the non-linear dark matter density field at every redshift, with a single amplitude, the bias $b$, that we fit to the data. The cross-correlation will be most sensitive to redshifts $z \sim 0.5-3$, with lower $z$ a poor match to CMB lensing, and higher $z$ not having substantial submm emission. As seen in the insets of Figure 3 . the $500 \mu \mathrm{m}$ emission is expected to have broader overlap with the CMB lensing kernel, and should therefore show a stronger correlation.
Fits are performed using points between $L=100$ and $L=1600$, as done in previous SPT lensing studies. The bestfit bias parameters for each observing wavelength and redshift distribution choice are shown in Table 2, with best-fit bias parameters depending on which redshift distribution is assumed. For the Béthermin et al. (2011) model we find $b \sim 1.8 \pm 0.3$ while the Viero et al. (2012) model for the CIB intensity gives $b \sim 1.3 \pm 0.2$. The uncertainties reflect statistical uncertainties only, and the large difference between the two models indicates that systematic uncertainties are substantial. The difference in bias factors is largely due to the different integrated mean intensities in the two models; for example, at $500 \mu \mathrm{m}$ the two models predict mean intensities that differ by a factor of 1.5, while the derived bias factors differ by a factor of 1.4. This difference in the mean intensity is larger than the $\sim 25 \%$ uncertainty in the FIRAS measurements (Fixsen et al.|1998); 
TABLE 2

FITS TO CONSTANT BIAS MODEL

\begin{tabular}{ccc} 
Wavelength & Bias (V12) & Bias (B11) \\
\hline $500 \mu \mathrm{m}$ & $1.29 \pm 0.16(12.6)$ & $1.80 \pm 0.22(12.7)$ \\
$350 \mu \mathrm{m}$ & $1.35 \pm 0.17(9.7)$ & $1.82 \pm 0.24(9.9)$ \\
$250 \mu \mathrm{m}$ & $1.34 \pm 0.23(11.8)$ & $1.56 \pm 0.27(12.0)$ \\
\hline
\end{tabular}

Cross-spectrum best-fit amplitudes to constant bias model for Viero et al (2012) (V12) and Bethermin et al. (2011) (B11) redshift distributions, $\chi^{2}$ of fit shown in parentheses. Quoted uncertainties only include statistical uncertainty.

the mean intensity in the Viero et al. (2012) model is more than $2 \sigma$ higher than that measured by FIRAS at $500 \mu \mathrm{m}$.

This simple model provides a very good fit, with $\chi^{2}=12.6$ or 12.7 for 14 degrees of freedom at $500 \mu \mathrm{m}$, depending on the assumed redshift distribution of the submm background. Despite the qualitative difference in the two redshift distributions apparent in the insets of Figure 3 good fits are obtained for both models, although a different normalization is preferred by each. This arises because most of the power is coming from the non-linear regime, where a power-law is a remarkably good fit to the clustering power spectra (Addison et al. 2012). As the cross-spectrum is a superposition of similar power-laws from different epochs, the detailed redshift distribution does not affect the shape of the cross-spectrum.

The bias factors at infrared and submm wavelengths have been measured using both source catalogs and auto power spectra of the diffuse backgrounds, as reviewed recently for the cosmic infrared background in Pénin et al. (2012). The inferred bias values depend on the assumed redshift distribution and intensity of the background, and the bias value that we measure is the clustering amplitude relative to the non-linear matter power spectrum, rather than either the linear matter power spectrum or a halo model, so a direct comparison is difficult. Using BLAST data at 500, 350, and $250 \mu \mathrm{m}$ (Viero et al.2009) and intensity estimates from Lagache et al. (2004), typical bias factors of $2.2 \pm 0.2$ were found (Pénin et al. 2012). Amblard et al. (2011) find slightly higher bias values using a halo model and fitting internally for the intensity as a function of redshift. The bias values found here are somewhat lower, but could be explained by differences in the assumed mean intensities and their redshift distributions.

Some studies of dusty sources at high redshift have led to substantially higher bias factors: Brodwin et al. (2008) found that $z \sim 2$ dusty, obscured galaxies selected in the optical/IR had bias factors $b \sim 3-5$, while Hickox et al. (2012) used sources selected at $870 \mu \mathrm{m}$ to estimate $b \sim 3$.

For comparison with lower redshift galaxy samples, recent results from SDSS-III find bias factors of $\sim 2$ for the massive galaxies (halo masses $\sim 5 \times 10^{13} h^{-1} M_{\odot}$ ) being targeted for baryon acoustic oscillation studies at $z \sim 0.3$ (Parejko et al. 2013), while bias estimates based on the SDSS main galaxy sample (McBride et al.2011) find $b=1-1.2$ for typical luminosity $\left(L_{*}\right)$. This suggests that the typical contributors to the submm background could be the higher redshift precursors to (or at least have the same mean bias as) galaxies that are intermediate in mass between these two samples.

In work that is closely related to the current work, Hildebrandt et al. (2013) cross-correlated gravitational lensing of
Lyman-break galaxies with a catalog of sources detected at $250 \mu \mathrm{m}$, and inferred typical masses of $1.5 \times 10^{13} M_{\odot}$ for these galaxies.

\section{DISCUSSION AND CONCLUSIONS}

We have shown that large-scale structure traced by submm sources is well-correlated with a CMB lensing convergence map. The cross-correlation is highly significant at 500, 350, and $250 \mu \mathrm{m}$, corresponding to detection significances of 8.9, 8.3 , and $6.7 \sigma$, respectively.

The cross-correlation between the lensing convergence map and each submm map is well fit by a simple constant bias model, with bias factors of $b=1.3-1.8$, depending on the assumed redshift distribution for the submm intensity. The lower bias factors are found for an assumed intensity distribution with more flux coming from higher redshifts.

There are several ways to extend the utility of the lensing convergence-SPIRE cross-power spectra presented here. For example, combining them with the cross-power and autopower spectra among the three SPIRE bands will probe the redshift distribution of the contributing sources and the correspondence between submm flux and the underlying dark matter distribution.

This technique is highly complementary to studies of the auto- and cross-correlations of submm background maps. While convergence maps have more noise (at current $\mathrm{CMB}$ map noise levels), concerns about Galactic cirrus or separating shot noise are greatly reduced, making cross correlation with $\mathrm{CMB}$ lensing an extremely robust probe of clustering with a promising future.

With the release of Planck maps covering a broad range of CIB wavelengths with well-matched angular resolution, it will be possible to perform a similar analysis over the entire 2500 square degree SPT survey area, while the coming Dark Energy Survey (DES) will also have nearly complete overlap with this area. DES will have both galaxy catalogs and cosmic shear maps with some resolution in the line of sight direction. In combination with the SPT CMB lensing convergence this will enable $3 \mathrm{D}$ mass maps of the universe extending to $z \sim 1100$.

The SPT is supported by the National Science Foundation through grant ANT-0638937, with partial support provided by NSF grant PHY-1125897, the Kavli Foundation, and the Gordon and Betty Moore Foundation. The McGill group acknowledges funding from the National Sciences and Engineering Research Council of Canada, Canada Research Chairs program, and the Canadian Institute for Advanced Research. Work at Harvard is supported by grant AST-1009012. S. Bhattacharya acknowledges support from NSF grant AST1009811, R. Keisler from NASA Hubble Fellowship grant HF-51275.01, B. Benson from a KICP Fellowship, M. Dobbs from an Alfred P. Sloan Research Fellowship, and O. Zahn from a BCCP fellowship.

\section{REFERENCES}

Addison, G. E., et al. 2012, ApJ, 752, 120

Amblard, A., et al. 2011, Nature, 470, 510

Béthermin, M., Dole, H., Lagache, G., Le Borgne, D., \& Penin, A. 2011, A\&A, 529, A4+
Bleem, L. E., et al. 2012, ApJ, 753, L9

Brodwin, M., et al. 2008, ApJ, 687, L65

Das, S., et al. 2011, Physical Review Letters, 107, 021301 

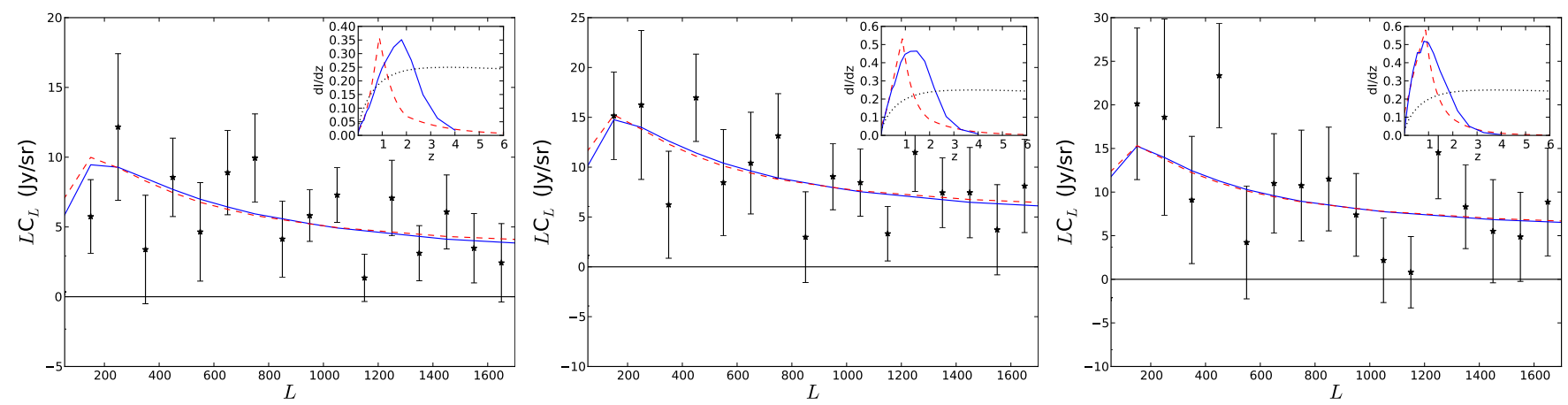

FIG. 3.- Cross-spectrum of lensing map and submm maps: left to right show 500, 350, $250 \mu \mathrm{m}$. Overplotted are best-fit constant bias models for two different redshift distributions for the submm intensity (with $d I / d z$ shown in inset in units of MJy/sr), with red (dashed) showing the model of Béthermin et al. (2011) and blue showing the model of Viero et al. (2012). Also shown in the inset, in arbitrary units, is the weighting of the CMB lensing kernel as a function of redshift (black dotted).

Fixsen, D. J., Dwek, E., Mather, J. C., Bennett, C. L., \& Shafer, R. A. 1998, ApJ, 508, 123

Griffin, M. J., Swinyard, B. M., \& Vigroux, L. G. 2003, in Presented at the Society of Photo-Optical Instrumentation Engineers (SPIE) Conference, Vol. 4850, IR Space Telescopes and Instruments. Edited by John C.

Mather . Proceedings of the SPIE, Volume 4850, pp. 686-697 (2003)., ed. J. C. Mather, 686-697

Hickox, R. C., et al. 2012, MNRAS, 421, 284

Hildebrandt, H., et al. 2013, MNRAS, 429, 3230

Hirata, C. M., Ho, S., Padmanabhan, N., Seljak, U., \& Bahcall, N. A. 2008,

Phys. Rev. D, 78, 043520

Hu, W. 2001, ApJ, 557, L79

Hu, W., \& Okamoto, T. 2002, ApJ, 574, 566

Lagache, G., et al. 2004, ApJS, 154, 112
Levenson, L., et al. 2010, MNRAS, 409, 83

McBride, C. K., Connolly, A. J., Gardner, J. P., Scranton, R., Scoccimarro,

R., Berlind, A. A., Marín, F., \& Schneider, D. P. 2011, ApJ, 739, 85

Parejko, J. K., et al. 2013, MNRAS, 429, 98

Pénin, A., Doré, O., Lagache, G., \& Béthermin, M. 2012, A\&A, 537, A137

Pilbratt, G. L., et al. 2010, A\&A, 518, L1

Schaffer, K. K., et al. 2011, ApJ, 743, 90

Sherwin, B. D., et al. 2012, Phys. Rev. D, 86, 083006

Smith, K. M., Zahn, O., \& Doré, O. 2007, Phys. Rev. D, 76, 043510

Song, Y.-S., Cooray, A., Knox, L., \& Zaldarriaga, M. 2003, ApJ, 590, 664

Story, K. T., et al. 2012, ArXiv e-prints, 1210.7231

van Engelen, A., et al. 2012, ApJ, 756, 142

Viero, M. P., et al. 2009, ApJ, 707, 1766

—. 2012, ArXiv e-prints, 1208.5049 